\title{
Interpelaciones identitarias en el trabajo: Propuesta para la comprensión de los procesos de construcción de la identidad laboral
}

\author{
Identitary interpellation in the workplace: Proposal \\ for understanding the processes of \\ identity construction at work
}

\author{
Álvaro SOTO \\ Antonio STECHER ${ }^{2}$ \\ Alan VALENZUELA
}

\begin{abstract}
Resumen
Se propone un modelo para analizar la relación entre la construcción narrativa de la identidad laboral y las dinámicas de interpelación identitaria en el mundo del trabajo. Se diferencian tres planos de interpelación - discursivo, estructural y de interacciones locales -, que pueden divergir o converger entre sí o en relación a la identidad para sí (heredada o anhelada) del sujeto. Hacer foco en las dinámicas de interpelación identitaria en el trabajo permite dar contexto y sentido al ejercicio reflexivo e interpretativo de construcción narrativa de la identidad en el trabajo. El modelo se ilustra a partir de dos estudios de caso de carácter cualitativo en Chile, uno con trabajadores de programas de acción pública del Estado y el otro con trabajadores de tiendas de la industria del retail. El modelo es aplicable teórica y metodológicamente en nuevos terrenos para ampliar la comprensión de las articulaciones entre trabajo y subjetividad en el mundo contemporáneo.
\end{abstract}

Palabras clave: Identidad; Interpelación; Narrativa; Trabajo; Subjetividad.

\footnotetext{
Abstract

This study proposes a model for analyzing the relationship between the narrative construction of work identity and the dynamics of identity interpellation in the world of work. Three levels of interpellation are identified - discursive,

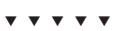

1 Universidad Alberto Hurtado, Facultad de Psicología. Almirante Barroso 10, Santiago de Chile, Chile. Dirección de correspondencia/ Correspondence to: A. SOTO.E-mail: <asoto@uahurtado.cl>.

2 Universidad Diego Portales, Facultad de Psicología. Santiago de Chile, Chile.

El texto es resultado de dos Proyectos de investigación "La construcción de identidades laborales en escenarios de trabajo heterogéneos: estudio en el sector público" (Proyecto Fondecyt n 1140602, Fondo Nacional de Desarrollo Científico y Tecnológico, Chile) y "Procesos de construcción de identidad en el trabajo en el Chile actual: el caso de los trabajadores de tiendas de grandes empresas del retail. Aportes empíricos y conceptuales al debate sobre trabajo e identidad en América Latina" (Proyecto Fondecyt n 11130095, Fondo Nacional de Desarrollo Científico y Tecnológico, Chile).
} 
structural, and local interactions -, which can diverge or converge with one another or in relation to the identity for itself (inherited or desired) of the subject. Focusing on the dynamics of identity interpellation at work allows the possibility to contextualize and give meaning to the reflective and interpretative exercise of the narrative construction of identity in the workplace. The model is illustrated by using two qualitative case studies conducted in Chile, one with workers participating in public action programs implemented by the State and the other with workers employed by large retail companies. The model is theoretically and methodologically applicable in new fields and can expand our understanding on the relationship between work and subjectivity in the contemporary world.

Keywords: Identity; Interpellation; Narrative; Work; Subjectivity.

El estudio de las identidades laborales, sus cambios y continuidades, en el contexto de las transformaciones tecno-socio-productivas del capitalismo contemporáneo, ocupa un lugar central en la actual agenda de investigación social en América Latina y a nivel mundial (Alvesson, 2010; Andrade, 2014; Gaete \& Soto, 2012; Pulido-Martínez, 2012; Ribeiro, 2012; Sennett, 2000; Sisto, 2012; Soto \& Gaete, 2013; Stecher, 2012). Desde diversas categorías analíticas, perspectivas teóricas y estrategias metodológicas se han explorado los procesos de construcción identitaria de los actores laborales, buscando ampliar la comprensión sobre las múltiples y emergentes articulaciones entre trabajo y subjetividad (Alvesson, Ashcraft, \& Thomas, 2008; Coutinho, Krawulski, \& Soares, 2007; Soto, 2012; Stecher, 2014; Tittoni \& Nardi, 2011).

Se entiende a las identidades laborales como producciones simbólicas articuladas narrativamente, centradas en la propia historia laboral, que expresan las modalidades específicas en que un trabajador interpreta y experimenta su contexto de trabajo (Stecher, 2012, 2013). A partir de dicha narrativa, el trabajador dota de continuidad, sentido y diferenciación su experiencia laboral, asignando particulares significados a la organización y a los otros con los que se relaciona (jefaturas, colegas, clientes, etc.), y definiéndose a sí mismo como un actor singular al interior de ese espacio social-laboral (Dubar, 1991, 1998, 2000; Soto, 2012; Stecher, 2012, 2013; Watson, 2008).

La producción de la identidad laboral supone la movilización de diferentes referentes simbólicos disponibles en el entorno socio-laboral (imaginarios, discursos, ideales de sujeto, etc.). Este fenómeno se da siempre en el marco de interacciones sociales constricciones institucionales, los modelos productivos y las asimetrías de poder y recursos en los espacios laborales, así como los trayectos biográficos y los anclajes socioestructurales de los actores laborales.

Como señala Dubar (1991), en el análisis de las identidades laborales es importante considerar tanto las modalidades de heterocategorización a partir de las cuales el contexto de trabajo (discursos, prácticas, materialidades) interpela y posiciona al trabajador en ciertos roles y categorías (identidad para otro), como los modos de autocategorización que el trabajador porta al ingresar a un espacio laboral y que ha ido construyendo a lo largo de su biografía (identidad para sí). Es en el marco de esas dinámicas, muchas veces múltiples y conflictivas, de heterocategorización y autocategorización, que los trabajadores elaboran narrativamente sus identidades laborales, las cuales deben ser pensadas en su carácter procesual, estando siempre abiertas a la impugnación, el cambio, la redefinición o modulación a partir de la capacidad de reflexividad e interpretación del sujeto (Duero, 2006; Soto, 2008; Stecher, 2013).

Conviene precisar que desde esta perspectiva el estudio de las identidades laborales - entendidas en su carácter narrativo, procesual y situado -, no es un estudio del modo como una identidad, ya previamente constituida del individuo, se expresaría en el ámbito laboral. No se trata por tanto de un estudio de "la identidad" (ya dada) "en" el trabajo (como mero espacio de expresión de la identidad), sino del modo en que cada trabajador en tanto actor social construye en su contexto de trabajo una cierta narrativa identitaria a partir de la cual se posiciona y dota de sentido su experiencia laboral. El concepto de identidad laboral se distancia así de lecturas 
psicologicistas que remitirían al despliegue de una identidad, previamente construida, en un espacio, exterior a esa identidad, que sería el trabajo. Se asume por el contrario que los procesos simbólicos de producción de sentido de uno mismo como trabajador son indesligables de las experiencias y contextos de trabajo. Por eso a lo largo del artículo utilizamos la noción de "identidades laborales" o procesos de construcción identitaria en el trabajo, evitando en el plano conceptual la noción de "identidad en el trabajo". Por cierto, como la definición propuesta de "identidad laboral" destaca, en los procesos de construcción de las identidades laborales también cumplen un papel las definiciones y sentidos de si mismo que el actor laboral porta producto de su particular socialización laboral y trayecto biográfico previo, las cuales son confrontadas, desafiadas o reforzadas por las formas de reconocimiento y categorización que experimenta en el espacio laboral. De este modo, el concepto de identidad laboral asumido se distancia también de visiones estructuralfuncionalistas que reducen la identidad del trabajador a un mero derivado mecánico de la posición o rol que este ocupa en un determinado organigrama o en una determinada categorización socio-ocupacional (Stecher, 2012, 2013).

No obstante situarse conceptualmente en el espacio de frontera entre el actor laboral (agencia) y su contexto de trabajo (estructura), el estudio psicosocial de la identidad en el trabajo ha tendido a sobredimensionar, conceptual y metodológicamente, el componente narrativo de los procesos identitarios. Esto ocurre en desmedro de la conceptualización y del análisis riguroso del contexto socio-laboral en que esa narrativa se produce (Stecher, 2013), y, especialmente, de las modalidades específicas en que dicho contexto incide en los procesos de construcción identitaria.

Asumiendo ese desafío, algunos trabajos en torno a la identidad laboral han resaltado la importancia de los procesos de interpelación al sujeto desde su entorno de trabajo (Bialakowsky, 2004; Du Gay, 2007; Longo, 2004; Sisto, 2014). Sin embargo, se han constatado evidentes vacíos respecto de la naturaleza y el alcance de esta noción.
Este artículo propone, discute e ilustra una noción de interpelación identitaria en el trabajo, buscando contribuir en la generación de herramientas analíticas útiles para pensar y estudiar empíricamente la relación entre el contexto de trabajo y la construcción narrativa de la identidad laboral, pertinente a las fuertes transformaciones experimentadas en los contextos de trabajo producto de los procesos de reestructuración productiva, flexibilización y modernización neoliberal en América Latina (De la Garza, 2000). Respecto de la interpelación identitaria, el artículo distingue tres planos en la que se despliega, e ilustra su potencial analítico para el estudio de las identidades laborales a partir de la presentación de dos estudios empíricos en Chile.

\section{La dinámica narración-interpelación en la construcción identitaria}

El concepto de interpelación, introducido inicialmente por Althusser en la tradición del marxismo estructural de la década de 1970, y posteriormente revisado y enriquecido por distintos autores, es una herramienta conceptual y analítica que permite avanzar en una mejor comprensión del modo específico en que los contextos de trabajo, a través de diferentes modos de categorizar y posicionar (interpelar) a los sujetos, inciden en las narrativas identitarias (identidades laborales) de los trabajadores. Althusser (1970/1988) propuso entender la interpelación como aquel mecanismo mediante el cual la ideología recluta sujetos entre los individuos a partir de un proceso simbólico indesligable de instituciones, prácticas y materialidades. El discurso ideológico interpela a los individuos concretos a partir del acto de representación en la cadena de significantes, y produce sujetos dispuestos a operar como soporte de las funciones requeridas por los diferentes niveles de la estructura social. El surgimiento del sujeto se basa en un reconocimiento instantáneo a través del cual el individuo interpelado se sabe convocado como sujeto de un particular tipo, consistente con el orden social del cual es parte. Como escribe Althusser en su clásico texto Ideología y Aparatos Ideológicos de Estado, 
... toda ideología tiene por función (función que la define) la constitución de los individuos concretos en sujetos... Sugerimos entonces que la ideología "actúa" o "funciona" de tal modo que "recluta" sujetos entre los individuos o transforma a los individuos en sujetos por medio de esta operación muy precisa que llamamos interpelación... (1970/1988, p.147).

La dinámica ideología-interpelación-producción de sujetos, en la obra de Althusser, está marcada por un énfasis determinista. En ella, la base económica de la vida social determina en última instancia a la superestructura política, jurídica e ideológica, y donde esta última, a partir de mecanismos de interpelación funcionales a dicha base y superestructura, produce a los individuos como un particular tipo de sujeto consistente con el orden social y sus formas vigentes de dominación.

Siguiendo y al mismo tiempo criticando la obra de Althusser, autores como Hall (2003) y Willis (1977/1981) han problematizado el carácter determinista de la noción de interpelación y el desconocimiento de los procesos de agenciamiento, reflexividad e interpretación a través de los cuales las interpelaciones pueden ser resignificadas o rechazadas por los actores sociales, individuales y colectivos, existiendo un constante juego de múltiples posicionamientos y reposicionamientos, tanto por las estructuras como por los propios actores. Para estos autores, la interpelación supone un proceso dinámico de negociación entre lo que el sujeto es y porta, producto de su biografía e historia y el contenido de ciertas interpelaciones en un contexto y momento dado.

Law (1999), por otra parte, propone una estrecha relación entre las dimensiones material e ideológica en los procesos de interpelación, que otorga a la noción un carácter más amplio que la operación de las palabras, abarcando esta también el modo en que los objetos, tecnologías y materialidades posicionan y disponen a los individuos en particulares modos de existir como sujetos. Law propone la imagen de un desorden de referencias, tendencias y posibilidades de ser, sugiriendo la necesidad de describir una ecología amplia, abierta y cambiante de las interpelaciones y de observar cómo éstas interfieren entre sí y cómo se manifiestan en las narrativas de los sujetos.

En base a este recorrido conceptual, es posible entender la interpelación identitaria en el trabajo como la serie de procesos y operaciones simbólicas, imbricadas siempre en instituciones, prácticas y materialidades concretas, que posicionan, definen e identifican al trabajador en una cierta categoría socio-laboral o modo de ser. Dichas interpelaciones pueden ser múltiples y diversas, se despliegan en diferentes planos del escenario de trabajo y pueden ser divergentes o convergentes entre sí. En el crisol de dichas interpelaciones, los actores laborales, agentes con capacidad de reflexividad y autointerpretación y portadores de una identidad para sí forjada en su trayecto biográfico y laboral previo, producen sus narrativas identitarias, las que dan cuenta de múltiples modalidades de apropiación, resignificación o rechazo de dichas interpelaciones que le son dirigidas desde su entorno laboral específico.

\section{Tres planos de interpelación identitaria en el trabajo}

Proponemos diferenciar tres planos del proceso de interpelación identitaria en el trabajo, elementos del contexto de trabajo que categorizan y posicionan al trabajador como un particular tipo de sujeto laboral. Los tres planos de interpelación operan conjuntamente, se imbrican entre sí (a veces coincidentemente y otras veces en tensión), contribuyendo al carácter dinámico y siempre inacabado del proceso de construcción identitaria en el trabajo.

La distinción de estos tres planos de interpelación es producto de un progresivo ejercicio de elucidación teórico-conceptual sobre los procesos de construcción identitaria en el trabajo que venimos desarrollando hace varios años como equipo de investigación, y el cual está estrechamente conectado a los desafíos de interpretación que el material empírico de distintas 
investigaciones nos ha ido planteando. No se trata, por tanto, de una distinción que emerge inductivamente de un análisis empírico específico, sino de un proceso de modelización y sistematización teórica, pero en estrecha vinculación a las exigencias analíticas planteadas por el material empírico de diferentes estudios sobre el tema.

\section{La interpelación identitaria discursiva}

Una primera fuente fundamental de interpelación identitaria para los trabajadores son el conjunto de discursos (textos escritos o discursos orales) sobre el trabajo, las empresas, las profesiones, los trabajadores, etc. que circulan en los contextos de trabajo, o más ampliamente en la esfera pública y los medios masivos de comunicación. Los discursos operan como bases de identificación, proyectos y códigos morales que se proponen al sujeto y que compiten entre sí (Watson, 2008).

La identidad laboral utiliza simultáneamente los discursos sociales o narrativas culturales disponibles y socialmente legitimados, al mismo tiempo que crea nuevas narrativas basadas en pequeñas historias, biografías y experiencias locales de los actores laborales (Watson, 2009). Es posible, de este modo, entender la identidad laboral como el encuentro entre el "decirse" a sí mismo y los discursos sociales que intentan definir e interpelar al trabajador, de lo que resultan disposiciones subjetivas a las que el individuo adhiere temporalmente y que ejercen como puntos de anclaje o suturas dentro del trabajo narrativo (Hall, 2003).

Las prácticas narrativas, asimismo, pueden entenderse como prácticas performativas (Smith \& Sparkes, 2008). En ellas, el sujeto hace uso de discursos culturalmente disponibles que lo posicionan en un contexto determinado y que orientan estratégicamente y legitiman sus formas de acción (Potter \& Wetherell, 1987).

\section{La interpelación identitaria estructural}

El segundo plano de interpelación identitaria en el trabajo corresponde a los diferentes aspectos socioinstitucionales y socioestructurales del contexto de trabajo que posicionan y categorizan al trabajador de un particular modo. En este nivel se incluyen dinámicas vinculadas al proceso de trabajo, las formas de empleo, las estrategias de gestión de recursos humanos, las relaciones laborales, el patrón tecnológico, las reglas, jerarquías, relaciones de poder y modelo productivo de la empresa u organización, todo lo cual anida, a su vez, en una trama mayor de instituciones externas: el mercado laboral, sector productivo, legislación laboral, redes nacionales y globales de producción y consumo, etc. (Stecher, 2013).

Soto (2015; Gaete \& Soto, 2012) propone la noción operacional de escenario del trabajo para dar cuenta de las configuraciones que resultan de la combinación de estrategias que desarrollan las empresas empleadoras respecto de sus diferentes grupos dotacionales. Dichas empresas interpelan a los sujetos sobre lo que son y por lo que valen a partir de las condiciones de empleo (formas de contratación y de pago), de la organización del trabajo (contenidos de los puestos en el marco de los procesos de trabajo) y de las eventuales prácticas de gestión de personas (formación, desempeño, carrera, desarrollo del compromiso, etc.).

Las condiciones de empleo y las prácticas de gestión permiten situar el tipo de vínculo que la organización propone a los trabajadores y la estabilidad de la relación en el tiempo, así como el estatus del actor dentro del sistema. Los procesos de trabajo y las tareas interpelan a los actores en la medida que la autonomía ofrecida y el control ejercido dan cuenta del aporte esperado, del estatus asignado y de las competencias que son reconocidas por la institución (Soto, 2015).

\section{La interpelación identitaria en las interacciones locales}

El tercer plano de interpelaciones al que se enfrentan los actores en el mundo del trabajo refiere a las interacciones cotidianas en los espacios de trabajo, donde los otros (colegas, clientes, jefaturas, gerencias) posicionan y categorizan permanentemente al sujeto dentro de la red de 
actores individuales y colectivos en juego, en el marco de culturas locales o de oficios, espacios de poder y conflicto. Las relaciones interpersonales en el trabajo se expresan a través de dinámicas de poder, conflicto y reconocimiento a partir de las cuales se construye la identidad en el trabajo (Sainsaulieu, 1977).

Esos modos de categorización que reciben los trabajadores en sus interacciones cotidianas llevan también las marcas de anclajes socioestructurales que exceden, al mismo tiempo que atraviesan, el mundo del trabajo (clase, género, etnia, generación). Ello se expresa en trayectorias desiguales en mercados del trabajo heterogéneos y desiguales.

El trabajo identitario se despliega en el marco de la interacción y la conversación organizacional cotidiana, donde, a partir de la interacción, los diferentes actores buscan posicionarse entre sí, estableciendo, manteniendo, cambiando o rechazando las diferentes posiciones identitarias (Mclnnes \& Corlett, 2012). Las interacciones con los demás ofrecen a los actores la posibilidad de confirmar sus narrativas identitarias, a partir de un proceso de autoverificación en las reacciones con los pares, superiores o subordinados con los cuales se está en relación (Down \& Reveley, 2009).

Bajo esta perspectiva, el ejercicio narrativo se hace codependiente de la acción en el espacio de trabajo, donde el actor despliega estratagemas de identidad laboral que contienen marcados elementos dramatúrgicos de presentación de sí mismo, que los demás pueden confirmar a través de sus respuestas. La actuación frente a los otros constituye un esfuerzo por mantener y encarnar determinadas normas definidas socialmente que pueden ser interpeladoras de actuaciones y de la construcción simbólica de la identidad (Goffman, 1959/2009).

\section{El dinamismo de los tres planos de interpelación identitaria en el trabajo}

Los tres planos de interpelación planteados despliegan paralelamente y son posibles de identificar a través del acercamiento empírico al mundo del trabajo. Ciertamente, la diferenciación conceptual propuesta esconde la permeable frontera existente entre los diferentes planos, pero se hace pertinente distinguirlos para profundizar acerca de las dinámicas de convergencia y divergencia en los procesos de interpelación narración en la construcción de identidades en el trabajo desde tres niveles de análisis.

En un primer nivel de análisis, es posible analizar la (in)consistencia interna de cada plano de interpelación identitaria en el trabajo. Esto está representado por contradicciones o convergencias de las interpelaciones vinculadas a los diferentes discursos disponibles, a los diferentes componentes de la estructura del trabajo-empleo y a las diferentes interacciones del actor laboral.

En un segundo nivel, la distinción de planos de interpelación permite situar el análisis en convergencias y divergencias entre los tres planos de interpelación. Esto puede darse ya sea por las fuentes o el contenido de la interpelación como por los diferentes momentos en que ocurre la interpelación identitaria.

En un tercer nivel de análisis, la diferenciación de planos de interpelación permite abordar de mejor manera las convergencias y tensiones entre las heterocategorizaciones múltiples que recaen sobre el actor laboral y la identidad para sí o autointerpretación del mismo. El proceso de construcción identitaria puede entenderse como una permanente transacción que busca afrontar las brechas entre la "identidad para otros o para-losdemás" y la "identidad para-sí" que el sujeto ha ido incorporando en su proceso biográfico, la cual a su vez puede estar tensionada internamente entre las "identidades heredadas" desde sus experiencias iniciales de socialización y su deseo de construir nuevas identidades en el futuro, o "identidades apuntadas" al interior de las instituciones (Dubar, 1991, 1998, 2000).

En estos tres niveles analíticos es posible identificar dinámicas de convergencia y divergencia vinculadas a la interpelación en el trabajo, frente a las cuales se despliega el ejercicio narrativo de 
construcción de la identidad, lo que configura el carácter dinámico de las identidades en el trabajo. A partir del análisis de convergencias y divergencias es posible aprehender de mejor manera el problema de la estabilidad o movilidad de las narrativas identitarias, en cuanto se pueden distinguir diferentes dinámicas temporales y condiciones de estabilidad.

La propuesta conceptual planteada en torno a los tres planos de interpelación identitaria en el trabajo plantea desafíos y oportunidades de orden metodológico en la comprensión de identidades laborales. Exige combinar un acercamiento narrativo a la identidad laboral con aproximaciones sistemáticas a las interpelaciones de naturaleza discursiva, estructural e interaccional en los espacios de trabajo. Dentro de este marco general, las posibilidades de diseño son amplias y variadas, como se intentará demostrar a continuación a partir de dos estudios empíricos diferentes. Los estudios dan cuenta de terrenos de estudio y diseños de investigación distintos, instrumentos y métodos de análisis diferentes, lo que da cuenta de la flexibilidad empírica que ofrece la noción de interpelación identitaria propuesta.

\section{Primer estudio: Procesos de construcción identitaria en la industria del retail}

El primer estudio tuvo como objetivo general describir y comprender los procesos de construcción de identidades laborales de trabajadores de tiendas de grandes y modernizadas empresas de la industria del retail en Santiago de Chile (Stecher, 2012). Dicha industria - grandes holdings empresariales cuasimonopólicos, que controlan altos porcentajes del comercio minorista y administran grandes cadenas de supermercados, tiendas por departamento y para el mejoramiento del hogar -, ha experimentado un profundo proceso de modernización y reestructuración productiva en las últimas dos décadas. Producto de ese proceso, la industria del retail se ha convertido en uno de los sectores más importantes de la economía chilena en términos de crecimiento (más de 2000 locales a lo largo del país), generación de empleo (sobre
260000 empleos directos), aporte al Producto Bruto Interno, creación de redes globales de comercio, innovación tecnológica y procesos de reorganización en que se articulan diversas estrategias de flexibilidad interna y externa, con lógicas de racionalización neotaylorista del trabajo (Calderón, 2006; Stecher, 2013).

\section{Diseño del estudio}

El estudio, desarrollado desde una perspectiva crítico-interpretativa (Thompson, 1993) y utilizando un enfoque narrativo de investigación (Lawler, 2002), realizó y analizó 72 entrevistas a trabajadores (gerentes, jefaturas intermedias, vendedores, cajeras, reponedores, operarios, personal de aseo y seguridad) de tiendas de departamentos, supermercados y tiendas de mejoramiento del hogar ubicadas en Santiago de Chile. A partir del análisis narrativo de los relatos de los trabajadores se construyó un modelo de 7 perfiles identitarios o tipos de identidad laboral, los cuales dan cuenta de modos prototípicos de narrar e interpretar la experiencia laboral dentro de las tiendas. Cada perfil identitario expresa una particular autocomprensión como trabajador del retail y un particular significado del trabajo en las tiendas, un específico ideal de trabajador y código moral, un modo singular de relacionarse a la empresa y de visualizar el futuro laboral, específicos colectivos de pertenencia y diferenciación, entre otros (Stecher, 2012).

Junto a las entrevistas narrativas se llevaron a cabo 6 entrevistas a informantes claves de la industria. Así mismo se llevó, a lo largo de los dos años del trabajo de campo (2014-2015), un cuaderno de campo en que se registraron distintas observaciones del funcionamiento de las tiendas. A partir de estas fuentes de información, analizadas siguiendo los principios de categorización y codificación de la Teoría Fundada (Glaser \& Strauss, 1967; Strauss \& Corbin, 1990), se pudo reconstruir el contexto de trabajo o escenario laboral de las tiendas así como, en el caso de los informantes claves vinculados a las gerencias de las empresas, tener acceso a los discursos gerenciales, de corte neomanagerial, que predominan en dicha industria. 
A partir de los resultados de los estudios previos, el foco y la originalidad de la reflexión en este artículo es el análisis del perfil identitario de oficio a la luz de los tres 3 ejes de interpelación identitaria distinguidos, algo no desarrollado en publicaciones previas.

\section{Perfiles identitarios e interpelaciones identitarias}

Uno de los perfiles identitarios reconstruidos fue denominado "identidad de oficio amenazada" (Stecher, 2012). Este perfil da cuenta del colectivo de trabajadores de las tiendas que narra e interpreta su trabajo en términos del ejercicio de un oficio en el cual se es experto (maestros panaderos, maestros carniceros, vendedores expertos, etc.), en el que se fue formado dentro de la industria por algún maestro, a partir de años de experiencia, y el cual exige conocer y adscribir al código moral. Estos trabajadores narran su experiencia laboral en las tiendas no como un "mero trabajo", sino como una larga historia de adquisición y ejercicio responsable de un saber-hacer experto que los distingue dentro de la tienda y que los hace merecedores de un especial reconocimiento.

Este perfil se vincula fuertemente a trabajadores insertos en secciones de mayor estatus, con mejor remuneración, habitualmente por encima de los 35 años y con largos años en la industria ejerciendo el oficio. Confluyen en este perfil identitario una autoidentificación (identidad para sí construida a lo largo de varios años en la industria) como representante de un oficio y vocación particular, junto a una heterocategorización (identidad para otro) recibida desde la gerencia, los clientes y los colegas que los sitúan históricamente dentro del retail - tanto en el plano de los discursos, el marco estructural de trabajo y empleo y las interacciones locales en la tienda -, como trabajadores de un particular y superior estatus en las tiendas.

En los relatos asociados a este perfil destaca la descripción detallada de las destrezas y habilidades del oficio y la permanente diferenciación

32 simbólica de aquellos otros colectivos de trabajadores de la tienda, especialmente de los trabajadores jóvenes, part time y con contratos temporales. Estos son vistos como aquellos trabajadores nuevos, que ganan el salario mínimo, que no tienen compromiso, responsabilidad ni vocación, que están de paso en las tiendas y que hacen su trabajo sin ninguna dedicación.

Uno de los aspectos distintivos de este perfil es la coexistencia de una satisfacción por el oficio que se ejerce y por ciertas formas de reconocimiento contractual y simbólica que se mantienen, junto a un sentimiento de fuerte temor frente a un conjunto de procesos de modernización y reestructuración en el modelo de negocio de la industria del retail que han supuesto una fuerte amenaza para ellos. Estas reorganizaciones, orientadas por la búsqueda de reducción de costos y mayor eficiencia, han supuesto un socavamiento progresivo de los soportes materiales y simbólicos de dicho perfil de oficio.

Entre las medidas que mayormente amenazan al perfil de oficio, que pueden entenderse como nuevas interpelaciones identitarias que entran en tensión con las construcciones identitarias existentes, destaca el despido de trabajadores antiguos especializados y su reemplazo por trabajadores jóvenes de baja calificación bajo modalidades de empleo más precarias, el reemplazo de la figura del vendedor experto o integral por cajeros y ordenadores con tareas específicas, acotadas y protocolizadas, la apuesta por lógicas de autoservicio y venta por internet que eliminan la importancia del vendedor y la protocolización neotaylorista de la venta que restringen la autonomía y reducen las tareas más calificadas (carniceros o panaderos que ya no cortan carne ni hacen pan pues los productos llegan ya listos desde los depósitos centrales). Otros factores a destacar son la alta exigencia de polifuncionalidad, que lleva a trabajadores expertos a hacer tareas ajenas a su oficio y la puesta en circulación de un discurso que interpela a los trabajadores como una masa homogénea de "colaboradores" polifuncionales, disciplinados y fuertemente identificados con la empresa y el negocio. 
La temporalidad de las narrativas identitarias se basa en una fuerte distinción entre un antiguo mundo del retail en Chile - en el que se conseguían buenos sueldos, se podía proyectar una carrera, se respetaba a los trabajadores, se les reconocía su oficio y se los valoraba, había un ambiente de empresa familiar y de camaradería -, y un nuevo mundo del retail de grandes e impersonales corporaciones que han introducido fuertes cambios - de corte tanto flexible como neotaylorista-, que amenazan fuertemente la integridad y continuidad del perfil identitario de oficio. Las actuales interpelaciones que recibe este colectivo de trabajadores del retail son múltiples, manifiestan divergencias entre sí (dentro de un mismo plano y entre los 3 planos) y entran en tensión con la identidad de oficio (identidad para sí) que los trabajadores de dicho perfil construyeron a lo largo de varios años en la industria. En términos más específicos, podemos destacar los siguientes aspectos:

En el plano de las interpelaciones discursivas se destaca el discurso de las empresas y de los medios masivos de comunicación relativo a las transformaciones de la industria del retail, a la desaparición de la antigua industria familiar y sus trabajadores y al surgimiento de un nuevo retail más moderno y competitivo, que apuesta por un nuevo perfil (menor calificación, alta rotación, polifuncionalidad, menor especialización, bajos salarios, trabajadores jóvenes) en su fuerza de trabajo. Este discurso circula fuertemente en el espacio de las tiendas y opera como un referente simbólico divergente en los procesos de construcción identitaria de los trabajadores más antiguos.

En el plano de las interpelaciones estructurales, se destaca el cambio en el proceso de trabajo de las tiendas, que supone tanto tendencias a la polifuncionalidad como a la protocolización neotaylorista, así como el cambio en los discursos y prácticas de las áreas de gestión de recursos humanos y el tipo de trabajador que construyen simbólicamente, así como el tránsito a contratos y condiciones de empleo más precarios tanto para los antiguos trabajadores como, especialmente, los trabajadores jóvenes que ingresan. Este cambio en el escenario de trabajo de las tiendas ha implicado una interpelación que desdibuja y socava las bases materiales y simbólicas de la histórica identidad de oficio dentro de la industria del retail, devolviendo al trabajador, por el contrario, la imagen y el mandato de ser un "colaborador" polifuncional, homogéneo, sin experticia, precarizado, de alta rotación, disciplinado y a bajo costo.

En el plano de las interpelaciones de las interacciones locales, por el contrario, es posible destacar el modo como aún los clientes, los compañeros y los mandos medios de las tiendas siguen entregando en las interacciones cotidianas un particular estatus, valor y reconocimiento a los trabajadores de oficio, quienes siguen siendo llamados "maestros" (aunque cada vez su trabajo se protocolice y polifuncionalice más) o convocados a resolver situaciones complejas con clientes difíciles (vendedores antiguos y con experiencia en el oficio y el producto). Estas diferentes interpelaciones que entran en tensión y disputa, entre sí y respecto a la identidad para sí de los trabajadores de oficio, constituyen parte del complejo campo de interpelaciones identitarias en que los trabajadores de oficio del retail construyen sus narrativas identitarias, buscando dotar de sentido, coherencia, pertenencia y continuidad sus experiencias laborales en el contexto laboral de una industria en transformación.

\section{Segundo estudio: Profesionales ejecutores de la acción pública del Estado}

El segundo estudio, actualmente en curso (Proyecto Fondecyt $n^{\circ} 1140602$ ), se lleva a cabo en dos programas públicos del Estado en Chile, que para su ejecución se articulan a través de alianzas público privadas, siguiendo las premisas del New Public Management (Hood, 1991; Salamon, 2000). Un programa está centrado en asegurar un servicio básico a la población en desventaja, y el otro en la habilitación social de población vulnerable. En torno a cada Programa se construyen redes complejas donde se encuentran actores públicos y privados, 
reguladas a partir de diferentes instrumentos de financiamiento, planificación, evaluación y rendición de cuentas. Atrás queda la figura del funcionario público con empleo protegido y surgen diferentes tipos de trabajadores - de empleador público y privado -, que se insertan en algún momento de cada proceso.

Entre estos diferentes actores, los profesionales que pertenecen a instituciones privadas que ejecutan programas fueron particularmente analizados pues permiten ilustrar la dinámica narrativa-interpelación identitaria en el trabajo. Estos profesionales entran en contacto directo con los usuarios de la acción pública, sus tareas son altamente prescritas y reguladas, los objetivos de su trabajo son detalladamente operacionalizados y son permanentemente controlados por funcionarios estatales. Al mismo tiempo, deben enfrentar las naturales contingencias y diferencias vinculadas a la realidad de los usuarios, lo que les exige ir adecuando el trabajo en el marco de las reglas y tiempos que rigen el sistema.

\section{Diseño del estudio}

En una primera fase se describió y analizó la red de actores de cada programa, que se construye a partir de diferentes instrumentos (Halpern, Lascoumes, \& Le Galès, 2014; Salamon, 2000) que estructuran y regulan a los actores de la acción pública. Para esto se analizaron los documentos oficiales de cada programa, se realizaron cerca de 20 entrevistas a actores claves de la red y se observaron instancias de trabajo. Se identificaron además los diferentes escenarios del trabajo (Soto, 2015) existentes en cada red público-privada, a fin de identificar las interpelaciones estructurales presentes en la situación de trabajo. Así también, se utilizaron herramientas analíticas de la Psicología discursiva (Potter \& Wetherell, 1987) para revisar documentos oficiales del programa y de sus instrumentos, identificando las figuras de trabajador esbozadas.

La segunda fase del estudio, en curso, se centra en estudios de caso con alrededor de 8 actores de cada Programa. Con cada participante se realizó una entrevista inicial en donde se preguntó por su trayectoria laboral y sus percepciones sobre los instrumentos más importantes que rigen su trabajo, a fin que se desplegara la construcción narrativa de su identidad en el trabajo. La información se analizó según los principios de la teoría fundada (Glaser \& Strauss, 1967; Strauss \& Corbin, 1990), con el objetivo de identificar ejes, tensiones y ajustes narrativos. Luego se realizaron dos momentos de observación bajo la propuesta etnográfica de dispositivos (Sisto \& Zelaya, 2013) en momentos claves vinculados a dos de los instrumentos que estructuran la red, información que se analizó buscando encontrar dinámicas de interpelación interpersonal.

Finalmente, se realizó una entrevista final de confrontación en la que se discutieron las observaciones y se cerró el proceso. En todo el proceso de análisis, el equipo de trabajo realizó procesos sistemáticos de triangulación del análisis de la información.

\section{Construcciones narrativas e interpelaciones en la acción pública}

Se ilustrará a continuación la relación entre ejercicio narrativo e interpelación identitaria en los profesionales que pertenecen a instituciones privadas que ejecutan programas. Fueron tomados dos casos individuales.

Carolina es una profesional de las ciencias sociales que trabaja en una consultora privada que ejecuta un programa de habilitación social. Es coordinadora de proyectos, lo que implica gestionar recursos, planes y personas en el marco de un estricto proceso de trabajo definido en las bases del programa que su institución se ha adjudicado. A pesar de haber desarrollado casi enteramente su trayectoria (9 años) vinculada a esta organización, nunca ha tenido un contrato formal de trabajo, sino que presta servicios independientes, con frecuentes lagunas de desempleo.

Oscar es también un profesional de las Ciencias Sociales, que se desempeña en la empresa concesionaria que administra un programa asociado al suministro de un bien básico para la población. 
Es el encargado de asegurar el buen funcionamiento de las organizaciones sociales vinculadas al programa y trabaja en equipos interdisciplinarios con profesionales asociados a los procesos técnicos del bien suministrado. En su trayectoria ha pasado por diversos trabajos en instituciones privadas que prestan servicios al Estado. En este trabajo está hace dos años, recibe un sueldo fijo y posee contrato de trabajo indefinido.

En ambos casos se observa una narrativa identitaria fuertemente centrada en la posesión de habilidades de trabajo de terreno, vinculadas al conocimiento del usuario y a las estrategias pertinentes para ajustar los instrumentos del programa de acción pública a las características y contingencias de cada situación. En ese marco general de construcción de una imagen de sí en el trabajo, fuertemente marcada por el dominio de las competencias de trabajo en terreno, se observan interesantes dinámicas de tensión respecto de las interpelaciones y de ajustes narrativos desplegados para sortearlas.

Carolina despliega en su narrativa una imagen de sí en el trabajo fuertemente marcada por sus competencias distintivas, vinculadas a sus destrezas para el trabajo en terreno con diferentes comunidades sociales, las que atribuye a su formación, a su experiencia profesional y al conocimiento del usuario, permitiéndole diferenciar desafíos y flexibilizar su trabajo, jugando al límite dentro de los márgenes que deja la estandarización y resultados del programa. La fuerza de su construcción identitaria en torno a sus destrezas en terreno coincide con las interpelaciones que se observan en los perfiles exigidos por el Estado a las consultoras y se refuerza en la amplia autonomía que dispone para adecuar los instrumentos a las contingencias de los usuarios. Por otro lado, Carolina destaca sus habilidades para la planificación y gestión de proyectos, las que parecen coincidir directamente con las interpelaciones discursivas vinculadas al principio de la eficiencia que gira en torno a las alianzas público privadas, así como a las múltiples demandas de gestión asociadas a los instrumentos que regulan el funcionamiento de la red institucional.
Al narrar su trayectoria y dar cuenta de quién es en el trabajo, llama la atención la ambigüedad con que Carolina alude a una búsqueda frustrada de nuevos trabajos que aseguren mejores condiciones de empleo, donde lo que finalmente prima es la enumeración de veces en que vuelve a la empresa consultora luego de lagunas de desempleo, aceptando los nuevos roles que le ofrecen en función de los nuevos proyectos adjudicados. Si bien las interpelaciones estructurales vinculadas a la situación de empleo inestable tensan la narrativa de Carolina, se observan dos ajustes narrativos que intentan sortear estas tensiones.

El primer ajuste es que su narrativa propone un trayecto laboral ascendente y estable, aludiendo a hitos y logros que van dando cuenta de un ascenso progresivo en la complejidad y la responsabilidad de los roles asumidos, donde finalmente construye un sentido de estabilidad en el trabajo que le llevan a diferenciarse de los "otros" que trabajan en condiciones inestables. El segundo ajuste narrativo observado refiere al vínculo subjetivo que Carolina establece con el Estado, en donde señala que en el trabajo de terreno se diluye la frontera entre lo estatal y lo privado, identificándose como trabajadora de "lo público".

La narrativa de Oscar también releva las competencias de trabajo en terreno con organizaciones sociales, vinculada a su experiencia en diferentes tipos de programas sociales durante su trayectoria desde instituciones privadas. De hecho, se autodefine como un intermediario de programas gubernamentales, que sabe afrontar la diversidad de situaciones a través de su creatividad y flexibilidad.

No obstante, esa imagen de sí parece secundaria respecto del pilar a través del cual narra su trayectoria, que es el cambio continuo de trabajos de diferente naturaleza, motivado por la búsqueda continua de condiciones más estables de empleo y de mejor remuneración. Las elecciones de su trayectoria no se vincularon al contenido del trabajo sino a mejorar las condiciones de empleo, y casualmente se le exigían competencias de trabajo en terreno con comunidades. De hecho, el actual trabajo lo escoge por ofrecerle contrato indefinido, 
y luego se percata que las exigencias del trabajo coincidían con sus competencias. Las competencias de trabajo en terreno aparecen entonces como un elemento secundario en la trayectoria, que hoy permite integrar su narrativa y ofrecer una imagen valiosa de sí mismo como trabajador, desestimando redirigir su trayectoria hacia trabajos de distinta naturaleza. La identidad laboral de Oscar está construida principalmente en torno a un proyecto personal (el teatro, su familia) compatible con el trabajo. No obstante, y sin buscarlo, surge una imagen de sí fortalecida en cuanto actor competente en el trabajo de terreno.

Esta integración narrativa es posible en el marco de la coincidencia entre las diferentes formas de interpelación en el trabajo. La construcción narrativa coincide con las interpelaciones identitarias de carácter discursivas asociadas al programa, que posicionan al profesional de la concesionaria como un agente técnico al servicio de las comunidades y del logro de objetivos. Así mismo, las interpelaciones estructurales definen espacios de trabajo de alta autonomía a fin de cumplir los propósitos finales asociados al suministro del servicio básico. Apelando a estos objetivos puede negociar con sus contrapartes y hacer ajustes a los procesos dentro de los estrictos límites formales que define el programa, respondiendo además a las expectativas de las comunidades y de sus contrapartes estatales.

\section{Discusión}

La propuesta del modelo analítico discutido para aproximarse a los procesos de construcción identitaria en el trabajo releva la importancia de las interpelaciones identitarias, en cuanto múltiples operaciones simbólicas de heterocategorización que recaen sobre el sujeto en los espacios de trabajo y lo posicionan como un particular tipo de trabajador. Las interpelaciones se despliegan en tres planos vinculados entre sí pero interesantes de distinguir, porque permiten identificar convergencias $\mathrm{y} / \mathrm{o}$ divergencias dentro de cada plano o entre planos que, al conjugarse, definirán distintos contenidos y fuerzas interpelatorias para el actor, las que deberá gestionar, considerando a su vez la identidad para sí, a partir del ejercicio narrativo de construcción identitaria.

En el primer plano de interpelación identitaria, los discursos operan como bases de identificación que los actores pueden utilizar, moldear o reinterpretar para definir sus propias (dis)posiciones frente al trabajo y a los otros. El interés es identificar y analizar las narrativas canónicas disponibles y circulantes, desde aquellas más genéricas que atraviesan amplios mundos del trabajo - véase por ejemplo las discusiones de estas últimas décadas en torno a la narrativa del sujeto managerial (Boltansky \& Chiapello, 1999; Sisto, 2014; Soto, 2011) o su cercana versión de emprendedor (Bröckling, 2015) -, hasta aquellos discursos institucionales locales como el discurso sobre el ejecutor de programas de acción pública o los nuevos discursos dominantes en el mundo del retail. Acercarse al plano discursivo exige herramientas analíticas y metodológicas que indaguen más allá de los discursos que el actor asujetado -, reproduce en su narrativa identitaria, así como también buscar, identificar y analizar los múltiples discursos sociales que se movilizan desde los sistemas de gestión, las normativas, los instrumentos de trabajo y las grandes consignas de la comunicación organizacional. La multiplicidad y fuerza de esos discursos definirá relaciones de convergencia o divergencia entre sí, con interpelaciones de los otros dos planos o con las identidades para-sí del sujeto.

El segundo plano de interpelación identitaria pone foco en las características estructurales en las cuales se ejerce el trabajo, en donde el contenido de las tareas, el uso de las tecnologías, las formas de control del trabajo, la autonomía y la amplitud de competencias exigidas posicionan al sujeto respecto de su importancia en el sistema. Del mismo modo, las prácticas de contratación y renta interpelan subjetivamente respecto del vínculo del actor con las instituciones, la estabilidad e importancia de éste. Este plano exige estrategias metodológicas que, más allá del relato del trabajador, permitan reconstruir detallada y sistemáticamente el marco estructural tecno-socioproductivo del escenario de trabajo bajo estudio. 
Lo descrito respecto a la transformación del proceso de trabajo, en la industria del retail y en el Estado, ilustran claramente la importancia de ese plano en la comprensión de la dinámica interpelacionesnarrativas identitarias en el mundo del trabajo, y ofrece un ejemplo fecundo de cómo conectar directamente en el análisis los procesos de reestructuración productiva del nuevo capitalismo con las emergentes modalidades de identidad laboral.

El tercer plano de las interpelaciones en la interacción del trabajo exige al investigador acercarse de manera directa a observar los espacios de trabajo, para analizar las formas locales cotidianas en que los actores interactúan, se posicionan y categorizan recíprocamente. Las transformaciones del trabajo contemporáneo hacen muy diversos y móviles los espacios de interacción de los actores en el trabajo, dado el surgimiento y masificación de formas de trabajo vinculadas a la producción inmaterial y a múltiples actores de control (De la Garza, 2009), a partir de formas flexibles centradas en la iniciativa y en la amplitud de tareas, el cambio permanente, el vuelco hacia el entorno, los vínculos flexibles y la generación de redes organizacionales que rompen con la idea de espacios laborales cerrados y estables (Soto, 2009). En esos vínculos e interacciones locales, como quedó ilustrado en los dos estudios presentados, es posible que los actores se propongan categorizaciones divergentes entre sí, o que éstas no converjan con los discursos dominantes o las dimensiones estructurales del trabajo o las identidades para si previamente consolidadas.

Este artículo ha propuesto que, para entender los procesos de construcción identitaria, debe combinarse una aproximación narrativa que permita el despliegue de la subjetividad de los actores y un acercamiento sustantivo a los diferentes planos de interpelación identitaria. Las formas posibles de acceder a las interpelaciones identitarias en el trabajo son múltiples, y será opción de cada investigador el definirlas en función de su terreno de estudio, las prioridades de su búsqueda y las perspectivas teóricas específicas que las sustentan.

Como desafío futuro, resulta importante pensar con mayor detalle el modo en que se articulan las interpelaciones del mundo laboral con las de otros mundos sociales que también recibe cotidianamente el trabajador (mundo familiar, sociabilidades territoriales, espacios políticos, mundos de ocio, etc.), las cuales pueden incidir fuertemente, a pesar de su carácter extralaboral, en las identidades laborales (Spink, 2011). Asimismo, y rescatando la intención original de la noción de interpelación, resulta fundamental avanzar en el análisis de las interpelaciones que se han ido volviendo hegemónicas en el capitalismo contemporáneo y que apuntan a la construcción de un sujeto laboral flexible y "funcional" a los procesos de producción, reproducción y dominación social. Finalmente, destacando el carácter procesual, dinámico y en disputa de los procesos identitarios, resulta importante visibilizar las diversas formas de agenciamiento, individual y colectivo, que en distintos contextos de trabajo resisten o resignifican los mandatos e interpelaciones dominantes y abren nuevas posibilidades y modos de existencia en los espacios laborales de América Latina.

\section{Colaboradores}

Todos los autores han contribuido en la revisión y propuesta conceptual, en diversas partes de los estudios empíricos revisados y en la redacción final del artículo.

\section{Referencias}

Althusser, L. (1988). Ideología y aparatos ideológicos de Estado: Freud y Lacan. Buenos Aires: Nueva Visión. (Publicada originalmente en 1970).

Alvesson, M. (2010). Self-doubters, strugglers, storytellers, surfers and others: Images of self-identities in organization studies. Human Relations, 63(2), 193-217. http://dx.doi.org/10.1177/00187267093 50372

Alvesson, M., Ashcraft, K. L., \& Thomas, R. (2008). Identity matters: Reflections on the construction of identity scholarship in organization studies. Organization, 15(1), 5-28. http://dx.doi.org/10.1177/13505084 07084426

Andrade, V. (2014). Identidad profesional y el mundo del trabajo contemporáneo. Reflexiones desde un resumen de caso. Athenea Digital, 14(2), 117-145. http://dx.doi.org/10.5565/rev/athenea.1143 
Bialakowsky, A. L. (2004). Identidades en el mundo del trabajo: Entre la implicación y la interpelación. En O. Battistini (Comp.), El trabajo frente al espejo. Continuidades y rupturas en los procesos de construcción identitaria de los trabajadores (pp.5-17). Buenos Aires: Prometeo.

Boltansky, L., \& Chiapello, E. (1999). Le nouvel esprit du capitalisme. Paris: Gallimard.

Bröckilng, U. (2015). El self emprendedor, sociología de una forma de subjetivación. Santiago: Ediciones Alberto Hurtado.

Calderón, A. (2006). El modelo de expansión de las grandes cadenas minoristas chilenas. Revista de la CEPAL, 90, 151-170. Recuperado el Enero 18, 2016 desde http://repositorio.cepal.org/bitstream/handle/ 11362/11143/090151170_es.pdf?sequence=1

Coutinho, M. C., Krawulski, E., \& Soares, D. H. P. (2007). Identidade e trabalho na conteporaneidade: repensando articulações possíveis. Psicologia \& Sociedade, 19(Esp.), 29-37. http://dx.doi.org/10.1590/s0102-718 22007000400006

De la Garza, E. (2000). Introducción: El papel del concepto de trabajo en la teoría social del siglo XX. En E. De la Garza (Coord.), Tratado Latinoamericano de sociología del trabajo (pp.15-35). México: Fondo de Cultura Económica.

De la Garza, E. (2009). Hacia un concepto ampliado de trabajo. En J. C. Neffa, E. De la Garza, \& L. Muñiz (Comps.), Trabajo, empleo, calificaciones profesionales, relaciones de trabajo e identidades laborales (pp.11-14). Buenos Aires: Clacso.

Down, S., \& Reveley, J. (2009). Between narration and interaction: Situating first-line supervisor identity work. Human Relations, 62(3), 379-401. http://dx.doi.org/ 10.1177/0018726708101043

Du Gay, P. (2007). Organizing identity: Persons and organizations after theory. London: Sage.

Dubar, C. (1991). La socialisation: Construction des identités sociales et professionnelles. Paris: Armand Colin.

Dubar, C. (1998). Trajetórias sociais e formas identitárias: alguns esclarecimentos conceituais e metodológicos. Educação \& Sociedade, 19(62), 13-30. http://dx.doi. org/10.1590/S0101-73301998000100002

Dubar, C. (2000). La crise des identités: L'interprétation d'une mutation. Paris: Presses Universitaires de France.

Duero, D. (2006). Relato autobiográfico e interpretación: Una concepción narrativa de la identidad personal. Athenea Digital, 9, 131-151. Recuperado el Enero 18, 2016, desde http://www.redalyc.org/articulo.oa?id=5 3700908

Gaete, T., \& Soto, Á. (2012). Esta es mi trayectoria, este es mi trabajo: Narrativas e identidad en el trabajo en Chile. Psykhe, 21(2), 47-59. http://dx.doi.org/10.7764/ psykhe.21.2.544
Glaser, B. G., \& Strauss, A. L. (1967). The discovery of grounded theory: Strategies for qualitative research. New York: Aldine.

Goffman, E. (2009). La presentación de la persona en la vida cotidiana. Buenos Aires: Amorrortu. (Publicada originalmente en 1959).

Hall, S. (2003). Introducción: ¿Quién necesita "Identidad"? En S. Hall \& P. Du Gay (Coords.), Cuestiones de identidad cultural (pp.13-39). Buenos Aires: Amorrortu.

Halpern, C., Lascoumes, P., \& Le Galès, P. (2014). L'Instrumentation de l'action publique. Paris: Presses de Sciences.

Hood, C. (1991). A public management for all season? Public Administration, 69(1), 3-19. http://dx.doi.org/ 10.1111/j.1467-9299.1991.tb00779.x

Law, J. (1999). Machinic pleasures and interpellations. United Kingdom: Lancaster University. Retrieved January 18, 2016, from http://www.lancaster.ac.uk/ fass/resources/sociology-online-papers/papers/lawmachinic-pleasures-and-interpellations.pdf

Lawler, S. (2002). Narrative in social research. In T. May (Ed.), Qualitative research in action (pp.242-258). London: Sage.

Longo, M. E. (2004). Los confines de la integración social. Trabajo e identidad en jóvenes pobres. En O. Battistini (Comp.), El trabajo frente al espejo. Continuidades y rupturas en los proceso de construcción identitaria de los trabajadores (pp.199-234). Buenos Aires: Prometeo.

Mclnnes, P., \& Corlett, S. (2012). Conversational identity work in everyday interaction. Scandinavian Journal of Management, 28(1), 27-38. http://dx.doi.org/10.10 16/j.scaman.2011.12.004

Potter, J., \& Wetherell, M. (1987). Discourse and social psychology: Beyond attitudes and behaviour. London: Sage.

Pulido-Martínez, H. (2012). La investigación sobre la identidad en, para y por el trabajo en América Latina, como ejercicio crítico acerca del mundo laboral. Psykhe, 21(2), 77-85. http://dx.doi.org/10.7764/psykhe.21.2. 546

Ribeiro, M. A. (2012). Las construcciones identitarias en el trabajo en la contemporaneidad: Retrato de un grupo de trabajadores de São Paulo (Brasil). Psykhe, 21(2), 61-75. http://dx.doi.org/10.7764/psykhe.21.2. 545

Sainsaulieu, R. (1977). L'identité au travail. Paris: Presses de la Fondation Nationale des Sciences Politiques.

Salamon, L. (2000). The new governance and the tools of public action: An introduction. Fordham Urban Law Journal, 28(5), 1611-1674. Retrieved January 15, 2016, from http://ir.lawnet.fordham.edu/cgi/view content.cgi? article $=2182 \&$ context $=u$ lj

Sennett, R. (2000). La corrosión del carácter. Barcelona: Anagrama. 
Sisto, V. (2012). Identidades desafiadas: Individualización, managerialismo y trabajo docente en el Chile actual. Psykhe, 21(2), 35-46. http://dx.doi.org/10.7764/ psykhe.21.2.542

Sisto, V. (2014). Identidades en disputa: Identidades laborales en el contexto de las actuales transformaciones en la gestión pública. En A. Stecher \& L. Godoy (Eds.), Transformaciones del trabajo, subjetividad e identidades, lecturas psicosociales desde Chile y América Latina (pp.299-322). Santiago: RIL Editors.

Sisto, V., \& Zelaya, V. (2013). La etnografía de dispositivos como herramienta de análisis y el estudio del managerialismo como práctica local. Universitas Psychologica, 12(4), 1345-1354. http://dx.doi.org/10. 11144/Javeriana.UPSY12-4.edha

Smith, B., \& Sparkes, A. C. (2008). Contrasting perspectives on narrating selves and identities: An invitation to dialogue. Qualitative Research, 8(1), 5-35. http://dx.doi.org/10.1177/1468794107085221

Soto, A. (2008). Flexibilidad y nuevas formas de identidad profesional. En A. Soto (Ed.) Flexibilidad laboral y subjetividades. Santiago: LOM.

Soto, A. (2009). Formas y tensiones de los procesos de individualización en el mundo del trabajo. Psicoperspectivas, 8(2), 102-119. Recuperado el Enero 15, 2016 desde http://www.psicoperspectivas.cl/ index.php/psicoperspectivas/article/view/80

Soto, A. (2011). Narrativas de profesionales chilenos sobre sus trayectorias laborales: La construcción de identidades en el trabajo. Psykhe, 20(1), 15-27. http:// dx.doi.org/10.4067/S0718-22282011000100002

Soto, A. (2012). Introducción a la sección especial la construcción de identidades en el trabajo en América Latina. Psykhe, 21(2), 3-7. http://dx.doi.org/10.7764/ psykhe.21.2.537

Soto, A. (2015). Escenarios del trabajo, una aproximación a la heterogeneidad del trabajo contemporáneo en Latinoamérica. Estudios Sociales, 51, 198-212. http:// dx.doi.org/10.7440/res51.2015.16

Soto, A., \& Gaete, T. (2013). Tensiones en la construcción identitaria individualizada en el trabajo flexible. Universitas Psychologica, 12(4), 1167-1180. http://dx. doi.org/10.11144/Javeriana.UPSY12-4.tcii

Spink, P. (2011). Whatever happened to work: From the centrality of shoes, ships and sealing-wax to the problems posed by flying pigs. Athenea Digital, 11(3),
3-24. Recuperado el Enero 15, 2016, desde http:// psicologiasocial.uab.es/athenea/index.php/athenea Digital/article/view-File/955/598

Stecher, A. (2012). Perfiles identitarios de trabajadores de grandes empresas del retail en Santiago de Chile: Aportes psicosociales a la comprensión de las identidades laborales. Psykhe (Santiago), 21(2), 9-20. http://dx.doi.org/10.7764/psykhe.21.2.538

Stecher, A. (2013). Un modelo crítico-interpretativo para el estudio de las identidades laborales. Contribuciones a la investigación psicosocial sobre trabajo y subjetividad en América Latina. Universitas Psychologica, 12(4), 1311-1324. http://dx.doi.org/10.11144/ Javeriana.UPSY12-4.mcie

Stecher, A. (2014). El campo de investigación sobre transformaciones del trabajo, identidades y subjetividad en la modernidad contemporánea. Apuntes desde Chile y América Latina. En A. Stecher \& L. Godoy (Eds.), Transformaciones del trabajo, subjetividad e identidades, lecturas psicosociales desde Chile y América Latina (pp.19-76). Santiago: RIL editores.

Strauss, A. L., \& Corbin, J. (1990). Basics of qualitative research: Grounded theory procedures and techniques. Newbury Park: Sage.

Thompson, J. B. (1993). Ideología y cultura moderna. México: UAM.

Tittoni, J., \& Nardi, E. (2011). Sujetividade e trabalho. En L. Holzmann \& A. Cattani (Orgs.), Dicionário de trabalho e tecnología (pp.375-378). Porto Alegre: Zouk Editora.

Watson, T. J. (2008). Managing identity: Identity work, personal predicaments and structural circumstances. Organization, 15(1), 121-143. http://dx.doi.org/10. $1177 / 1350508407084488$

Watson, T. J. (2009). Narrative, life story and manager identity: A case study in autobiographical identity work. Human Relations, 62(3), 425-452. http://dx.doi. org/10.1177/0018726708101044

Willis, P. E. (1981). Learning to labor: How working class kids get working class jobs. New York: Columbia University Press. (Original work published 1977).

Recibido: Febrero 2, 2016

Versión final: Abril 29, 2016

Aprobado: Junio 14, 2016 
